\title{
Aptidão Física Relacionada à Saúde em Escolares de Jequié, BA, Brasil
}

\author{
Health-Related Physical Fitness in Students from Jequié, BA, Brazil
}

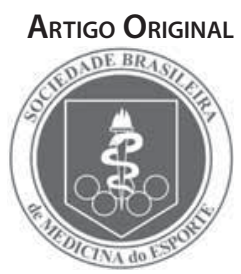

Valfredo Dórea ${ }^{1,2}$

Enio Ricardo Vaz Ronque ${ }^{2,3}$ Edilson Serpeloni Cyrino 2,3 Helio Serassuelo Junior 2,3 Luís Alberto Gobbo $2,3,4$ Ferdinando Oliveira Carvalho 2,3,4 Carine Ferreira de Souza² Juliana Cordeiro de Melo² Patrícia Aparecida Gaion²

1. Departamento de Educação Física. Universidade Estadual do Sudoeste da Bahia.

2. Grupo de Estudo e Pesquisa em Metabolismo, Nutrição e Exercício. Centro de Educação Física e Esporte. Universidade Estadual de Londrina.

3. Grupo de Estudo e Pesquisa em Atividade Física e Exercício. Centro de Educação Física e Esporte. Universidade Estadual de Londrina. 4. Programa de Pós-Graduação em Saúde Pública. Universidade de São Paulo.

5. Programa de Pós-Graduação em Educação Física. Universidade Católica de Brasília.

\section{Endereço para correspondência:} Edilson Serpeloni Cyrino. Grupo de Estudo e Pesquisa em Metabolismo Nutrição e Exercício. Centro de Educação Física e Esporte. Universidade Estadual de Londrina. Rod. Celso Garcia Cid, km 380, Campus Universitário - 86051-990

- Londrina, PR - Brasil.

E-mail: emcyrino@uel.br

Submetido em 29/07/2006

Versão final recebida em 10/09/2006 Aceito em 12/09/2006

\begin{abstract}
RESUMO
O objetivo deste estudo foi analisar a aptidão física relacionada à saúde (AFRS) em escolares de Jequié, BA, Brasil. Para tanto, a AFRS de 182 meninos e 160 meninas (sete a 12 anos), escolares da rede pública de ensino, foi avaliada mediante a aplicação dos testes motores corrida/caminhada de nove minutos (indicador de resistência cardiorrespiratória), abdominal modificado em um minuto (indicador de força e resistência abdominal), sentar-e-alcançar (indicador de flexibilidade). O somatório das espessuras das dobras cutâneas tricipital e subescapular foi utilizado como indicador de gordura corporal. Os resultados encontrados indicaram maior prevalência de meninos e meninas abaixo (19\% e 49\%, respectivamente) do que acima (3\% e 1\%, respectivamente) dos critérios estabelecidos pela AAHPERD (1988) para indicadores de gordura corporal. Por outro lado, somente $51 \%$ dos meninos e $58 \%$ das meninas no sentar-e-alcançar; $11 \%$ e $7 \%$, respectivamente, no abdominal modificado em um minuto, e 15\% e 14\%, respectivamente, na corrida/caminhada de nove minutos alcançaram os critérios estabelecidos. Portanto, os resultados sugerem que programas efetivos de intervenção parecem necessários para a promoção de mudanças no estado nutricional e na atividade física habitual de escolares de Jequié.
\end{abstract}

Palavras-chave: aptidão física, desempenho motor, testes motores, adiposidade corporal, crianças.

\begin{abstract}
The aim of this study was to analyze the health-related physical fitness (HRPF) in students from Jequié, BA, Brazil. Therefore, the HRPF of 182 boys and 160 girls aged 7-12 years, students from public schools, was assessed by the application of 9-minute-run/walk (indicator of cardiorespiratory capacity), modified in one minute abdominal (indicator of abdominal strength and endurance), sit-and-reach (indicator of flexibility) motor tests. The sum of the triciptal and subscapular skinfolds thickness was used as indicator of body fat. The results found indicated higher prevalence of boys and girls below (19\% and 49\%, respectively) than above (3\% and $1 \%$, respectively) the criteria established by the AAHPERD (1988) for indicators of body fat. Conversely, only $51 \%$ of the boys and $58 \%$ of the girls in the sit-and-reach; $11 \%$ and $7 \%$, respectively, in the modified in one minute abdominal; and $15 \%$ and $14 \%$, respectively, in the 9-minute-run/walk reached the established criteria. Thus, the results suggest that effective intervention programs seem necessary for the promotion of alterations in the nutritional status as well as habitual physical activity of students from Jequié.
\end{abstract}

Keywords: physical fitness, motor performance, motor tests, body adiposity, children.

\section{INTRODUÇÃO}

Atualmente, muitos estudos têm indicado que níveis satisfatórios de aptidão física relacionada à saúde podem favorecer a prevenção, manutenção e melhoria da capacidade funcional; reduzir a probabilidade do desenvolvimento de inúmeras disfunções de caráter crônico-degenerativas, tais como obesidade, diabetes, doenças cardiovasculares, hipertensão, dentre outras, proporcionando, assim, melhores condições de saúde e qualidade de vida à população(1-4).

Desse modo, a manutenção de níveis satisfatórios de aptidão física relacionada à saúde tem sido recomendada para indivíduos de ambos os sexos, em diferentes faixas etárias ${ }^{(5)}$. Todavia, maior ênfase tem sido dada nos períodos da infância e adolescência, uma vez que nessas fases da vida o organismo parece mais sensível às modificações relacionadas aos aspectos motores e da composição corporal(6).
As variáveis que melhor representam as características de crescimento físico, composição corporal e desempenho motor podem ser afetadas tanto por aspectos biológicos, quanto por fatores socioeconômicos e culturais, podendo exercer importantes influências sobre os níveis de saúde e qualidade de vida, sobretudo de crianças e adolescentes ${ }^{(7-9)}$.

Embora informações associadas ao comportamento de indicadores da composição corporal e aptidão física em escolares brasileiros atraiam o interesse de vários pesquisadores e profissionais das diversas áreas da saúde, a maioria dos dados previamente publicados tem-se restringido à análise do perfil de populações residentes nas regiões Sul e Sudeste do país. A escassez de pesquisas em outras regiões, sobretudo Norte-Nordeste, reduz o impacto sobre a necessidade de revisão das estratégias de políticas públicas e sociais a serem adotadas em diferentes municípios, Estados e regiões do Brasil. 
Portanto, investigações sobre o comportamento de indicadores da aptidão física relacionada à saúde em populações jovens, de diferentes estratos socioeconômicos, em diferentes regiões, podem fornecer valiosas informações para análise do estilo de vida adotado em diferentes sociedades, em diferentes períodos da história. Além disso, as informações produzidas podem possibilitar previsões para o futuro, principalmente no que tange aos aspectos relacionados à promoção da saúde e ao controle de doenças ${ }^{(10)}$.

Com base nessas informações, o objetivo do presente estudo foi analisar os níveis de aptidão física relacionada à saúde em escolares de sete a 12 anos, de Jequié, BA, Brasil.

\section{MÉTODOS}

\section{Local e população do estudo}

O município de Jequié, localizado às margens do Rio das Contas, no Sudoeste do Estado da Bahia, segundo dados divulgados pelo IBGE (http://www.ibge.com.br/censo), a partir do último censo populacional, ocupa a sexta posição em termos demográficos no Estado, contando com população estimada em 147.202 habitantes, situando-se atrás apenas de Salvador, Feira de Santana, Vitória da Conquista, Itabuna e Ilhéus. No momento em que foi desenvolvida a coleta de dados para o presente estudo, a população escolar de Jequié era composta por cerca de 26.500 jovens (crianças e adolescentes), todos regularmente matriculados entre o ensino pré-escolar e médio, nas redes municipal, estadual e particular de ensino, segundo dados fornecidos pelo Censo Escolar realizado pela Secretaria Estadual da Educação (http://www. sec.ba.gov.br).

\section{Cálculo amostral}

Extraiu-se aleatoriamente uma subamostra de um conjunto de 3.019 escolares, participantes de um projeto de pesquisa mais amplo sobre o perfil de indicadores de saúde, realizado nas escolas do município de Jequié. Quanto à escolha dos escolares por estabelecimento, utilizou-se o processo de aleatoriedade simples, resguardando alguns pontos considerados de extrema relevância para o estudo, tais como: idade, sexo, organização administrativa da escola, aquiescência dos pais mediante a autorização expedida pelo supervisor da pesquisa. Para o cálculo do tamanho da amostra foi adotado alfa de 95\% e poder de $80 \%$, com erro amostral de $2 \%$.

\section{Sujeitos}

A amostra para este estudo foi composta por 342 escolares (182 meninos e 160 meninas) na faixa etária de sete a 12 anos de idade, regularmente matriculados em escolas públicas do município de Jequié, BA, Brasil. A tabela 1 apresenta a distribuição da amostra de acordo com sexo e idade.

Tabela 1. Distribuição da amostra total de acordo com o gênero e a idade

\begin{tabular}{c|c|c|c}
\hline Idade (anos) & Meninos & Meninas & Total \\
\hline 7 & 28 & 35 & 63 \\
\hline 8 & 30 & 30 & 60 \\
\hline 9 & 22 & 33 & 55 \\
\hline 10 & 33 & 19 & 52 \\
\hline 11 & 32 & 21 & 53 \\
\hline 12 & 37 & 22 & 59 \\
\hline Total & 182 & 160 & 342 \\
\hline
\end{tabular}

Todos os responsáveis pelos escolares, após informados sobre o propósito desta investigação e os procedimentos a serem adotados, assinaram um termo de consentimento livre e esclarecido. Este estudo foi desenvolvido em conformidade com as instruções contidas na Resolução 196/96 do Conselho Nacional de Saúde para estudos com seres humanos, do Ministério da Saúde.

\section{Antropometria}

A massa corporal foi obtida em uma balança de plataforma com precisão de $0,1 \mathrm{~kg}$ e a estatura foi determinada em um estadiômetro de madeira com precisão de $0,1 \mathrm{~cm}$, de acordo com os procedimentos descritos por Gordon et al.(11). A partir dessas medidas calculouse o índice de massa corpórea (IMC) por meio do quociente massa corporal/estatura², sendo a massa corporal expressa em quilogramas $(\mathrm{kg})$ e a estatura em metros (m). As informações produzidas a partir do IMC foram utilizadas no presente estudo como indicador do estado nutricional, de acordo com as curvas de crescimento publicadas pelo $\mathrm{NCHS}^{(12)}$.

A composição corporal foi avaliada pela técnica de espessura de dobras cutâneas. Três medidas foram tomadas em cada ponto, em seqüência rotacional, do lado direito do corpo, sendo registrado o valor mediano. Para tanto, foram aferidas as espessuras das dobras cutâneas subescapular e tricipital. As medidas foram realizadas por um único avaliador com um adipômetro científico da marca Cescorf, com precisão de $0,1 \mathrm{~mm}$ e pressão constante em sua abertura de $10 \mathrm{~g} / \mathrm{mm}^{2}$, de acordo com as técnicas descritas por Slaughter et al. ${ }^{(13)}$. O somatório das espessuras de dobras cutâneas foi utilizado como indicador de gordura subcutânea, seguindo os valores referenciais propostos pela AAHPERD(14).

\section{Testes motores}

Uma bateria de testes motores foi aplicada obedecendo à seguinte ordem seqüencial: sentar-e-alcançar (SA), abdominal modificado em um minuto (ABD) e corrida/caminhada de nove minutos (9MIN), seguindo as padronizações descritas pela AAHPERD(14). Para a análise quanto ao atendimento dos aspectos relacionados à saúde foram utilizados os critérios sugeridos pelo Physical Best ${ }^{(14)}$.

Todos os testes foram aplicados nos estabelecimentos de ensino, exceto o teste de $9 \mathrm{MIN}$, que foi realizado no estádio municipal, por ser o único local que possui pista de atletismo com as recomendações oficiais.

\section{Tratamento estatístico}

Os dados foram tratados inicialmente por procedimentos descritivos de média e desvio-padrão. Tabela de percentis, com base na distribuição de freqüências do IMC, foi estabelecida para um diagnóstico do estado nutricional da amostra. A normalidade dos dados foi confirmada pelo teste de Kolmogorov-Smirnov. Assim, para a comparação entre grupos etários e sexos foi empregada análise de variância por dois fatores (Anova two-way). O teste post hoc de Scheffé, para comparações múltiplas, foi utilizado para a identificação das diferenças entre as médias. Distribuição de freqüência relativa foi adotada para verificar a proporção de escolares que atenderam aos critérios de saúde. Para as comparações entre duas proporções foi aplicado o teste de significância para diferenças entre proporções. $O$ nível de significância estabelecido para as análises foi de $P<0,05$. As informações foram processadas no pacote computacional Statistica ${ }^{\text {tm }}$, versão 5.1 . 


\section{RESULTADOS}

A distribuição dos valores do IMC em intervalos de percentis é apresentada na tabela 2. Verificou-se relativamente alta prevalência de crianças com risco de desnutrição (valores $<10^{\circ} \mathrm{P}$ ), tanto nos meninos ( 20\%) quanto nas meninas ( 10\%). Por outro lado, a prevalência de crianças com risco de obesidade (valores $\geq 90 \circ$ ) foi reduzida, tanto em meninos ( $2 \%$ ) quanto em meninas (<1\%).

A tabela 3 apresenta os valores de massa corporal, estatura e IMC dos escolares investigados, de acordo com sexo e idade cronológica. Interação entre sexo e idade foi encontrada somente na variável massa corporal $(P<0,01)$. O efeito isolado do sexo foi verificado tanto para a massa corporal $(P<0,01)$ quanto para o IMC $(P<0,05)$. Por outro lado, um efeito isolado da idade foi identificado em todas as variáveis analisadas $(P<0,01)$, apresentando tanto os meninos quanto as meninas valores crescentes com o avançar da idade.

Tabela 2. Distribuição de freqüências do IMC de crianças e adolescentes de acordo com os intervalos de percentis do CDC-NCHS

\begin{tabular}{c|c|c|c|c|c}
\hline \multirow{2}{*}{ IMC } & \multicolumn{2}{|c|}{ Meninos } & \multicolumn{2}{c|}{ Meninas } & \multirow{2}{*}{ Total } \\
\cline { 2 - 5 } & $\mathbf{n}$ & $\%$ & $\mathbf{n}$ & $\%$ & \\
\hline$<3 \circ \mathrm{P}$ & 12 & 6,6 & 4 & 2,5 & 16 \\
\hline $3 \circ \mathrm{P}+10 \circ \mathrm{P}$ & 24 & 13,2 & 12 & 7,5 & 36 \\
\hline $10 \circ \mathrm{P}+50 \circ \mathrm{P}$ & 83 & 45,6 & 84 & 52,5 & 167 \\
\hline $50 \circ \mathrm{P}+90 \circ \mathrm{P}$ & 59 & 32,4 & 59 & 36,9 & 118 \\
\hline $90 \circ \mathrm{P}+97 \circ \mathrm{P}$ & 4 & 2,2 & 1 & 0,6 & 5 \\
\hline$>97 \circ \mathrm{P}$ & 0 & 0 & 0 & 0 & 0 \\
\hline Total & 182 & 100 & 160 & 100 & 342 \\
\hline
\end{tabular}

Tabela 3. Valores médios ( \pm DP) de massa corporal, estatura e IMC em escolares de Jequié/BA, de acordo com o gênero e a idade

\begin{tabular}{|c|c|c|c|c|c|c|}
\hline \multirow{2}{*}{$\begin{array}{l}\text { Idade } \\
\text { (anos) }\end{array}$} & \multicolumn{2}{|c|}{ Massa corporal (kg) } & \multicolumn{2}{|c|}{ Estatura $(\mathrm{cm})$} & \multicolumn{2}{|c|}{ IMC $\left(\mathrm{kg} / \mathrm{m}^{2}\right)$} \\
\hline & Meninos & Meninas & Meninos & Meninas & Meninos & Meninas \\
\hline \multirow{2}{*}{7} & 21,3 & 21,2 & 120,6 & 120,8 & 14,5 & 14,5 \\
\hline & $\pm 2,5$ & $\pm 2,1$ & $\pm 5,0$ & $\pm 4,5$ & $\pm 1,0$ & $\pm 1,1$ \\
\hline \multirow{2}{*}{8} & 25,2 & 25,3 & 126,7 & 126,3 & 15,7 & 15,8 \\
\hline & $\pm 2,1$ & $\pm 2,1$ & $\pm 3,7$ & $\pm 3,9$ & $\pm 1,0$ & $\pm 1,0$ \\
\hline \multirow{2}{*}{9} & 28,3 & 28,5 & 130,5 & 132,0 & 16,6 & 16,3 \\
\hline & $\pm 2,9$ & $\pm 2,9$ & $\pm 4,0$ & $\pm 3,2$ & $\pm 1,5$ & $\pm 1,6$ \\
\hline \multirow{2}{*}{10} & 30,4 & 31,2 & 137,6 & 138,3 & 16,0 & 16,2 \\
\hline & $\pm 3,8$ & $\pm 4,9$ & $\pm 6,5$ & $\pm 7,0$ & $\pm 1,5$ & $\pm 1,7$ \\
\hline \multirow{2}{*}{11} & 32,8 & 35,9 & 140,7 & 141,5 & 16,6 & 17,9 \\
\hline & $\pm 3,7$ & $\pm 5,3$ & $\pm 4,8$ & $\pm 6,9$ & $\pm 2,0$ & $\pm 2,2$ \\
\hline \multirow{2}{*}{12} & 35,7 & 39,7 & 143,9 & 146,9 & 17,3 & 18,3 \\
\hline & $\pm 3,8$ & $\pm 6,6$ & $\pm 6,6$ & $\pm 7,8$ & $\pm 2,3$ & $\pm 2,3$ \\
\hline Fsexo & \multicolumn{2}{|c|}{$10,91^{* *}$} & \multicolumn{2}{|c|}{2,50} & \multicolumn{2}{|c|}{$4,38^{*}$} \\
\hline Fidade & \multicolumn{2}{|c|}{$155,15^{* *}$} & \multicolumn{2}{|c|}{$168,40^{* *}$} & \multicolumn{2}{|c|}{$27,19^{* *}$} \\
\hline Fsexo $x$ idade & \multicolumn{2}{|c|}{$3,09^{* *}$} & \multicolumn{2}{|c|}{0,60} & \multicolumn{2}{|c|}{1,77} \\
\hline
\end{tabular}

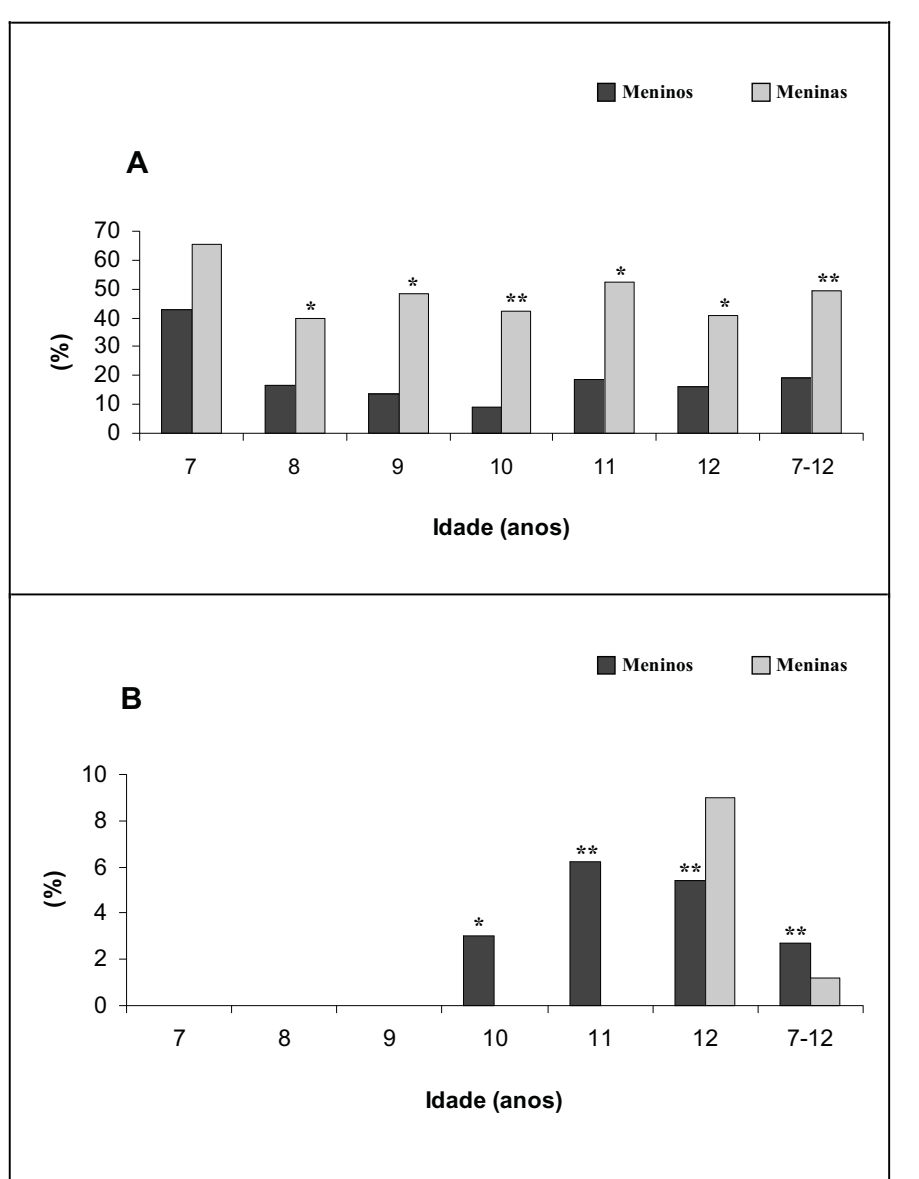

Figura 1 - Proporção (\%) de escolares situados abaixo (A) e acima (B) dos critérios de saúde estabelecidos para o somatório das espessuras de dobras cutâneas tricipital e subescapular

${ }^{* *} P \leq 0,01$ e ${ }^{*} 0,01<P<0,05$.

As proporções (\%) de escolares situados abaixo (1A) e acima (1B) dos critérios estabelecidos pela proposta do Physical Best ${ }^{(14)}$ para o somatório de espessuras de dobras cutâneas, de acordo com o sexo e a idade, são apresentadas na figura 1. Verificou-se maior proporção de escolares que ficaram aquém do limite inferior dos critérios de saúde estabelecidos em relação aos que se situaram acima deles, em ambos os sexos. Além disso, diferenças estatisticamente significantes entre os sexos foram identificadas nas proporções de escolares que se enquadraram abaixo dos critérios estabelecidos, com exceção da idade de sete anos $(P=0,07)$. Por outro lado, para os escolares que se situaram acima dos critérios, diferenças significativas foram identificadas aos 10 anos $(P=0,01)$, aos 11 anos $(P=0,0001)$, 12 anos $(P=$ $0,006)$ e no conjunto de todas as idades $(P=0,0006)$. Os resultados demonstraram que por volta de $78 \%$ dos meninos e $50 \%$ das meninas atenderam aos critérios estabelecidos para espessuras de dobras cutâneas. Entretanto, o que mais chama a atenção é a elevada prevalência de escolares situados abaixo dos critérios estabelecidos, sobretudo entre as meninas (49\%).

Na figura 2 é apresentado o percentual de escolares que alcançaram os critérios estabelecidos para cada um dos testes motores investigados. Em relação ao teste de SA, um número maior de meninas (58\%) atingiu os critérios estabelecidos em comparação aos meninos (51\%). Apesar disso, nenhuma diferença estatisticamente significante foi encontrada entre os sexos, em nenhuma faixa etária analisada, bem como no conjunto de todas as idades $(P=0,23)$. Em relação à 


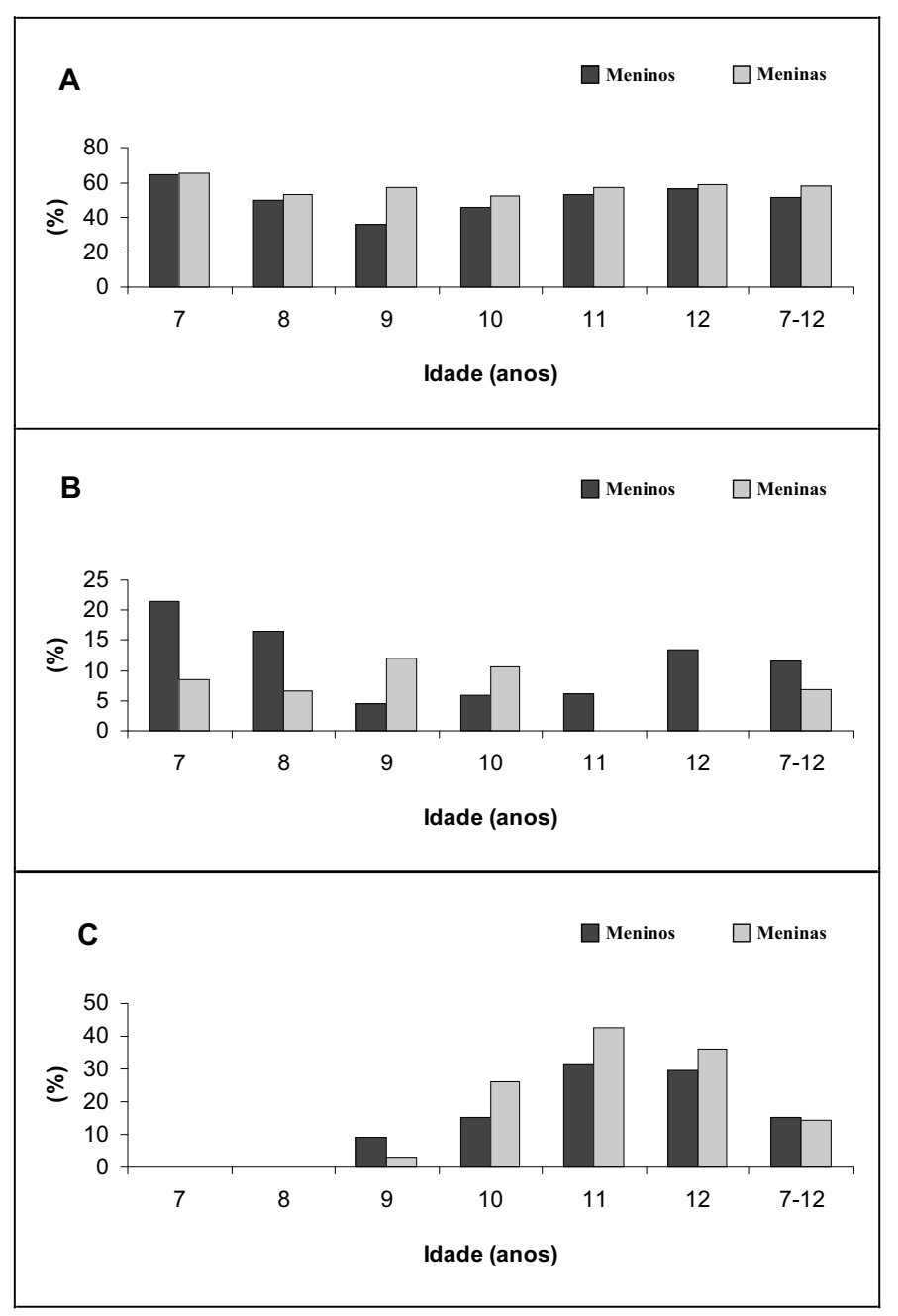

Figura 2 - Proporção (\%) de escolares que alcançaram os critérios de saúde estabelecidos para resultados de testes motores. (A) sentar-e-alcançar; (B) abdominal modificado em um minuto; (C) corrida/caminhada de nove minutos.

Nota. Nenhuma diferença significante foi encontrada entre os sexos nas comparações entre sujeitos de mesma idade $(P>0,05)$.

idade observou-se que em ambos os sexos houve estabilidade na proporção de escolares que alcançaram os valores recomendados (figura 2A).

Por outro lado, no teste ABD (figura 2B) apenas um número reduzido de escolares, de ambos os sexos, atendeu aos critérios propostos. Vale destacar que em algumas idades houve casos de meninas que não conseguiram realizar sequer uma única repetição. Analisando-se o conjunto de todas as idades, verificou-se que somente $11 \%$ dos meninos e $7 \%$ das meninas alcançaram o número de repetições mínimas proposto pelo referencial adotado pelo presente estudo. Nenhuma diferença estatisticamente significativa foi encontrada nas comparações entre as proporções $(P>0,05)$.

A melhora no teste de desempenho observada no decorrer da idade no teste de 9MIN começou a ser observada a partir dos nove anos, em ambos os sexos. Entretanto, com base na análise do conjunto de todas as idades, verificou-se que apenas uma pequena proporção de escolares (15\% dos meninos e 14\% das meninas) conseguiu atender aos critérios estabelecidos pela AAHPERD ${ }^{(13)}$, sem diferenciação estatística entre os sexos $(P=0,80)$. O que chama a atenção é que nas idades de sete e oito anos nenhum dos escolares investigados conseguiu atingir o critério mínimo proposto (figura 2C).

\section{DISCUSSÃO}

A importância de investigar índices de aptidão física relacionada à saúde fundamenta-se na tentativa do estabelecimento de padrões desejáveis em relação ao desempenho motor e a gordura corporal que, quando atingidos, possam assegurar algum tipo de proteção contra o surgimento e o desenvolvimento de disfunções de caráter hipocinético(15).

Com base nas informações produzidas pelo indicador de adiposidade utilizado, verificou-se que aproximadamente 18\% dos meninos e 40\% das meninas apresentaram quantidade de gordura corporal acima dos critérios adotados na presente investigação, enquanto somente cerca de 10\% dos meninos e 7\% das meninas demonstraram valores abaixo dos valores recomendados. Esses dados são preocupantes, uma vez que o excesso de gordura corporal está associado com o aumento na incidência de diversas disfunções crônico-degenerativas ${ }^{(16,17)}$, ao passo que quantidades mínimas de tecido adiposo (wasting) também podem induzir inúmeros distúrbios metabólicos, potencializando o aumento da incidência e a gravidade de enfermidades infecciosas, contribuindo assim para o aumento das taxas de mortalidade infantil, bem como para retardos no desenvolvimento psicomotor $^{(18,19)}$

Vale lembrar que a prevalência da desnutrição no Brasil, apresenta forte associação com a região geográfica; nas regiões Norte e Nordeste, a desnutrição é pelo menos duas vezes maior do que na região Centro-Oeste e quatro vezes maior que na região Sul ${ }^{(20)}$. Entretanto, esse fenômeno parece estar, por via de regra, atrelado às variações das condições socioeconômicas e culturais da amostra investigada.

Nesse sentido, Guedes \& Guedes ${ }^{(21)}$ verificaram comportamento semelhante ao observado no presente estudo, em escolares de escolas públicas de Londrina (PR). Por outro lado, em estudo recentemente realizado nesse mesmo município, com uma amostra de elevado nível socioeconômico (classes média alta e alta), foi identificado comportamento bastante distinto, ou seja, aproximadamente $7 \%$ dos meninos e $15 \%$ das meninas apresentaram quantidades de gordura corporal abaixo do critério preconizados para a saúde ${ }^{(14)}$, enquanto cerca de 33\% e $15 \%$, respectivamente, dos meninos e das meninas demonstraram valores acima desses mesmos critérios ${ }^{(10)}$

O aumento constatado no decorrer dos anos no número de meninas com valores de adiposidade corporal acima dos critérios propostos para saúde pode ser explicado, pelo menos em parte, pelo processo maturacional(6). Entretanto, o excesso de gordura corporal na infância e na adolescência pode estar relacionado, também, com os aspectos nutricionais, econômicos e culturais.

Nesse sentido, Abrantes et al. ${ }^{(22)}$ relataram que a região Sudeste tem proporção maior de crianças e adolescentes com sobrepeso e obesidade do que a região Nordeste. Esses dados foram confirmados posteriormente por Magalhães \& Mendonça ${ }^{(23)}$, em estudo conduzido com adolescentes.

Vale ressaltar que diversos fatores, tais como a elevada escolaridade e renda familiar e a presença de aparelhos de televisão, computadores, vídeogames e telefones nas residências, parecem ter associação positiva e significante com a presença de sobrepeso e obesidade. Esses fatos foram constatados por Oliveira et al. ${ }^{(24)}$, em estudo realizado com escolares pertencentes a escolas públicas e privadas, de cinco a nove anos, no município de Feira de Santana (BA). Outro achado relevante desse estudo foi o fato de que os alunos de escolas privadas demonstraram ter 2,5 mais probabilidade de ser obesos do que seus pares de escolas públicas. 
Ao contrário do presente estudo que identificou, em 18\% dos meninos e 40\% das meninas investigadas em Jequié (BA), quantidades de gordura corporal abaixo dos critérios recomendados, Ronque et al.(25), em uma amostra proveniente de escola privada de Londrina, na faixa etária de sete a 10 anos, classificada como sendo de alto nível socioeconômico, verificaram prevalência de sobrepeso ( 19\%) e obesidade $(\sim 14 \%)$ bastante superior à da média da população brasileira de mesma faixa etária.

Portanto, os programas de intervenção a serem propostos para a realidade brasileira não devem desprezar as condições extremas encontradas no país, uma vez que em $11 \%$ das famílias brasileiras coexistem condições de baixo peso e sobrepeso; 45\% das famílias com pelo menos um membro com baixo peso também têm um membro com sobrepeso ${ }^{(26)}$.

Com relação ao comportamento de indicadores funcionais e neuromotores, há indícios da existência de forte associação entre níveis satisfatórios de desempenho motor e o bom funcionamento orgânico. Desse modo, possuir níveis adequados de resistência cardiorrespiratória e aptidão física musculoesquelética (força/resistência e flexibilidade) pode conduzir a diminuição na incidência de fatores de risco para muitas disfunções de caráter crônico-degenerativo(27).

Nesse sentido, no presente estudo, as meninas apresentaram melhores resultados no teste de SA do que os meninos, o que também foi verificado por Ronque(10), em escolares de sete a 10 anos de idade, do município de Londrina (PR) e por Gaya et al. ${ }^{(28)}$, em escolares de sete a 15 anos de idade, do município de Porto Alegre (RS). A redução nos valores do teste de $S A$, verificada nos meninos aos 10 anos de idade neste estudo, pode estar relacionada ao surto de crescimento pré-púbere, no qual os ossos longos apresentam índice de crescimento longitudinal mais acentuado do que os músculos e os tendões ${ }^{(6)}$.

Por outro lado, no teste $A B D$ verificou-se maior prevalência de crianças abaixo dos critérios (93\% de meninos e 97\% de meninas) do que a encontrada por Guedes \& Guedes ${ }^{(21)}$ (53\% de meninos e 48\% de meninas), em escolares de escola pública de Londrina (PR), e por Ronque et al. ${ }^{(10)}$ com escolares de alto nível socioeconômico no mesmo município (23\% de meninos e $27 \%$ de meninas). Esse fato provavelmente possa ser explicado, pelo menos em parte, pelos hábitos de atividade física e pelo estado nutricional de moradores dessa região do país. Essas variáveis, contudo, merecem ser melhor investigadas, uma vez que não foram controladas no presente estudo.

Para corroborar essa hipótese, estudo conduzido por Prista et al. ${ }^{(7)}$ em Maputo, Moçambique, com crianças residentes em diferentes regiões socioeconômicas, indicou menor desempenho em testes motores que envolviam resistência e força muscular em crianças pertencentes a regiões menos favorecidas economicamente.

No presente estudo, o aumento observado nos escores do teste $9 \mathrm{MIN}$ com o avançar da idade, tanto nos meninos quanto nas meninas, provavelmente seja produto dos processos de maturação, crescimento e desenvolvimento ${ }^{(6)}$. Apesar disso, a baixa proporção de escolares que atenderam aos critérios utilizados causa preocupação, uma vez que estudos têm indicado a existência de uma relação inversa entre os índices de resistência cardiorrespiratória e o desenvolvimento de fatores de risco para doenças crônico-degenerativas ${ }^{(29,30)}$.

Apesar de os valores encontrados em Jequié serem inferiores aos apresentados por Ronque et al. ${ }^{(10)}$ e por Guedes \& Guedes ${ }^{(21)}$, verificouse redução das diferenças entre as populações no decorrer das faixas etárias, sobretudo entre as meninas. Essa melhora no desempenho com o avançar da idade também foi verificada por Guedes \& Guedes ${ }^{(21)}$, em escolares de ambos os sexos, levando-nos a acreditar que, dentre os componentes da aptidão física relacionada à saúde, a amostra de Jequié apresenta comportamento mais próximo ao de crianças das regiões Sul do país na aptidão cardiorrespiratória.

Vale ressaltar que o referencial adotado em todas as análises, no presente estudo, foi construído com base em dados obtidos na população dos Estados Unidos, um país desenvolvido e com características socioeconômicas e culturais bastante diferenciadas da maior parte da realidade brasileira.

Além disso, as informações deste estudo foram obtidas mediante uma série de medidas e testagens, provavelmente desconhecidas até aquele momento por muitos dos sujeitos investigados, o que, no caso dos testes motores, principalmente, pode repercutir desfavoravelmente, uma vez que a familiarização prévia ou a simples repetição da tarefa motora a ser desempenhada pode resultar em melhoria do desempenho motor, devido ao incremento da capacidade do sistema neural em ativar totalmente a musculatura, a melhor coordenação dos músculos sinergistas e antagonistas ou, ainda, a melhoria da coordenação do movimento ${ }^{(31)}$.

As limitações apresentadas anteriormente sugerem que os dados produzidos por este estudo sejam analisados com cautela. Nesse sentido, as comparações com dados produzidos por outros estudos, sobretudo, conduzidos em diferentes regiões do Brasil, possibilita a ampliação do nível de análise e das possíveis inferências.

\section{CONCLUSÕES}

Os resultados do presente estudo indicaram maior prevalência de baixos índices de adiposidade corporal do que índices elevados, tanto nos meninos, quanto nas meninas, sugerindo um quadro mais próximo da desnutrição do que de sobrepeso ou obesidade.

Os indicadores de desempenho motor utilizados demonstraram que a grande maioria dos escolares investigados apresentou índices inferiores aos pontos de corte sugeridos para uma aptidão física satisfatória pelo Physical Best ${ }^{(14)}$, para crianças de ambos os sexos, na faixa etária estudada.

Por outro lado, apesar dos resultados desfavoráveis observados no teste de 9MIN, os índices de aptidão cardiorrespiratória apresentados pelos escolares de Jequié foram aqueles que mais se assemelharam aos valores encontrados em outros estudos conduzidos com escolares, nas regiões Sul e Sudeste do Brasil.

As informações produzidas pela presente investigação sinalizam a necessidade de novos estudos que se proponham a controlar variáveis importantes, tais como níveis de atividade física habitual, hábitos alimentares, níveis socioeconômicos, além de experiência prévia dos sujeitos com as tarefas motoras a serem investigadas. Acredita-se que, desse modo, seria possível explicar em grande parte o comportamento observado nos jovens de Jequié/BA, o que poderia favorecer, sobremaneira, o estabelecimento de ações de intervenção voltadas para a melhoria dos níveis de saúde e da qualidade de vida dessa população, tanto no presente quanto no futuro.

\section{AGRADECIMENTOS}

Os autores agradecem ao CNPq e a Capes pelas bolsas concedidas.

Todos os autores declararam não haver qualquer potencial conflito de interesses referente a este artigo. 


\section{REFERÊNCIAS BIBLIOGRÁFICAS}

1. Boreham C, Riddoch C. The physical activity, fitness and health of children. J Sports Sci 2001;19:915-29.

2. Morris JN. Exercise in the prevention of coronary heart disease: today's best buy in public health. Med Sci Sports Exerc 1994;26:807-14.

3. Morton BGS, Taylor WC, Snider SA, Huang IW, Fulton JE. Observed levels of elementary and middle school children's physical activity during physical education classes. Prev Med 1994;3:437-441.

4. Tammelin T, Näyhä S, Laitinen J, Rintamäki H, Järvelin MR. Physical activity and social status in adolescence as predictors of physical inactivity in adulthood. Prev Med 2003;37:375-81.

5. Böhme MTS. Aptidão física: aspectos teóricos. Rev Paul Educ Fís 1993;7:52-68.

6. Malina RM, Bouchard C, Bar-Or O. Growth, maturation and physical activity. 2nd ed. Champaign: Human Kinetics Books, 2004

7. Prista A, Marques A, Maia J. Relationship between physical activity, socioeconomic status and physical fitness of 8-15-year-old youth from Mozambique. Am J Hum Biol 1997;9:449-57.

8. Ronque ERV. Crescimento físico e aptidão física relacionada à saúde em escolares de alto nível socioeconômico. 2003. Dissertação (Mestrado em Educação Física) - Faculdade de Educação Física Universidade Estadual de Campinas, Campinas, 2003.

9. Serassuelo Jr H. Análise das variáveis de crescimento, composição corporal e desempenho moto em escolares de diferentes níveis socioeconômicos da cidade de Cambé - Paraná. 2002. Dissertação (Mestrado em Educação Física) - Escola de Educação Física e Esporte, Universidade de São Paulo, São Paulo, 2002.

10. Ronque ERV, Cyrino ES, Dórea VR, Serassuelo Jr H, Galdi EHG, Arruda M. Diagnóstico da aptidão física em escolares de alto nível socioeconômico: avaliação referenciada por critérios de saúde. Rev Bras Med Esporte 2006;12:prelo.

11. Gordon CC, Chumlea WC, Roche AF. Stature, recumbent length, and weight. In: Lohman TG, Roche AF, Martorel R, editors. Anthropometric standardization reference manual. Champaign, IL: Human Kinetics, 1988;3-8

12. CDC (Centers for Disease Control and Prevention), 2002. 2000 CDC Growth Charts for the United States: Methods and Development. Vital Health Statistics Series 11. Washington, DC: U.S. Government Printing Office.

13. Slaughter MH, Lohman TG, Boileau RA, Stillman RJ, Van Loan M, Horswill CA, et al. Influence of maturation on relationship of skinfolds to body density: a cross-sectional study. Hum Biol 1984;56:681-9.

14. AAHPERD. Physical Best. Reston, VA: American Alliance for Health, Physical Education, Recreation and Dance, 1988

15. Sallis JF, Simons-Morton BG, Stone EJ, Corbin CB, Epstein LH, Faucette N, et al. Determinants of physical activity and intervention in youth. Med Sci Sports Exerc 1992;24:248-57s.
16. Ball GDC, MacCargar $L$ J. Childhood obesity in Canada: a review of prevalence estimates and risk factors for cardiovascular diseases and type 2 diabetes. Can J Appl Physiol 2003;28:117-40.

17. Hooper CA, Gruber MB, Munoz KD, Macconnie SE, Pfingston YM, Nguyen K. Relationship of blood cholesterol to body composition, physical fitness, and dietary intake measures in third-grade children and their parents. Res Q Exerc Sport 2001;72:182-8

18. Bjorntorp P. Classification of obese patients and complications related to the distribution of surplus fat. Am J Clin Nutr 1987;45:1120-5.

19. Monteiro CA, Conde WL. Tendência secular da desnutrição e da obesidade na infância na cidade de São Paulo (1974-1996). Rev Saúde Públ 2000;34:52-61.

20. Oliveira O, Taddei, JAAC. Efeito dos vieses de sobrevivência nas prevalências da desnutrição em crianças no sexto ano de vida. Brasil-PNSN. Cad Saúde Públ 1998;14:493-9.

21. Guedes DP, Guedes JERP. Aptidão física relacionada à saúde de crianças e adolescentes: avaliação referenciada por critério. Rev Bras Ativ Fís Saúde 1995;1:27-38.

22. Abrantes MM, Lamounier JA, Colosimo EA. Prevalência de sobrepeso e obesidade em crianças e adolescentes das regiões Sudeste e Nordeste do Brasil. J Pediatr (Rio J) 2002;78:335-40.

23. Magalhães VC, Mendonça GAS. Prevalência e fatores associados a sobrepeso e obesidade em adolescentes de 15 a 19 anos das regiões Nordeste e Sudeste do Brasil, 1996 a 1997. Cad Saúde Públ 2003;19:129-39.

24. Oliveira AMA, Cerqueira EMM, Souza JS, Oliveira AC. Sobrepeso e obesidade infantil: influência de fatores biológicos e ambientais em Feira de Santana, BA. Arq Bras Endocrinol Metab 2003;47:144-50.

25. Ronque ERV, Cyrino ES, Dórea VR, Serassuelo Jr H, Galdi EHG, Arruda M. Prevalência de sobrepeso e obesidade em escolares de alto nível socioeconômico em Londrina, Paraná, Brasil. Rev Nutr 2005:18:709-17.

26. Doak CM, Adair LS, Monteiro C, Popkin BM. Overweight and underweight coexist within households in Brazil, China and Russia. J Nutr 2000;130:2965-71.

27. Pate RR, Pratt M, Blair SN, Haskell WL, Macera CA, Bouchard C, et al. Physical activity and public health. A recommendation from the Centers for Disease Control and Prevention and the American College of Sports Medicine. JAMA 1995;273:402-7.

28. Gaya A, Cardoso M, Siqueira O, Torres L. Crescimento e desempenho motor em escolares de 7 a 15 anos provenientes de famílias de baixa renda. Rev Mov 1997:4:1-23.

29. Carnethon MR, Gidding SS, Nehgme R, Sidney S, Jacobs DR, Liu K. Cardiorespiratory fitness in young adulthood and the development of cardiovascular disease risk factors. JAMA 2003;290:3092-100.

30. Ross R, Katzmarzyk PT. Cardiorespiratory fitness is associated with diminished total and abdominal obesity independent of body mass index. Int J Obes Relat Metab Disord 2003;27:204-10.

31. Van Praagh E, Dore E. Short-term muscle power during growth and maturation. Sports Med 2002:32:701-28. 